\title{
Cytomegalovirus Infection and Pre-Eclampsia
}

\author{
Rădulescu Carmen ${ }^{*}$, Huțanu Adina², Gabor Rozalia ${ }^{3}$, Șincu Nina ${ }^{4}$ \\ 1 Department of Obstetrics and Gynecology II, University of Medicine and Pharmacy of Tirgu.Mureș, Romania \\ 2 Department of Central Laboratory, Emergency University Hospital, Tirgu Mureș, Romania \\ 3 Department of Management and Economy, University Petru Maior of Tirgu Mureș, Romania \\ 4 Department of Infectious Diseases, University of Medicine and Pharmacy of Tirgu Mureș, Romania
}

\begin{abstract}
Introduction: Pre-eclampsia is a pregnancy-specific disease characterized by hypertension after 20 weeks of gestation and proteinuria. It is a major cause of maternal and perinatal morbidity and mortality. The pathogenesis of pre-eclampsia is not completely understood. In our study we investigated if there is a potential link between cytomegalovirus infection and pre-eclampsia and if cytomegalovirus infection is the triggering factor of pre-eclampsia. Material and methods: This study was carried out in a secondary care hospital between January 2014 and July 2015. We included two groups of pregnant women: 66 with pre-eclampsia and 62 without pre-eclampsia. Enzyme linked immunosorbent assay (ELISA) technique was performed to detect cytomegalovirus Immunoglobulin G levels in maternal serum. Results: The p-value between median values of positive cytomegalovirus IgG in pre-eclamptic women and in controls was not significant ( $p$ :0.867). Odds ratio for cytomegalovirus IgG in pre-eclamptic group (OR:0.967; 95\% Cl:0.535-1.748) do not differ greatly from the value recorded in the control group (OR:1.036; 95\% Cl:0.571-1.880). The risk for preterm birth in pre-eclamptic women with cytomegalovirus lgG positive values (OR:1.009; 95\%Cl:0.329-3.090) was greater than those which had term delivery (OR:0.994; 95\%Cl:0.472-2.095) but the two values are not very high. We found a positive correlation coefficient $(0.217)$ at a "p" value of 0.08 between white blood cells, but a negative correlation coefficient between the percentage of neutrophils and cytomegalovirus infection. Conclusion: Cytomegalovirus infection may be associated with pre-eclampsia but it is unlikely to be the triggering factor of pre-eclampsia.
\end{abstract}

Keywords: Cytomegalovirus infection (CMV), pre-eclampsia (PE), Enzyme linked immunosorbent assay (ELISA), Immunoglobulin G (IgG), Interleukine (IL)

Received: 30 January 2016 / Accepted: 22 March 2016

\section{Introduction}

Pre-eclampsia $(\mathrm{PE})$ is an important cause of maternal and perinatal morbidity and mortality. It is a pregnancy-specific disease characterized by high blood pressure: 140/90 $\mathrm{mmHg}$ or more taken twice a day more than 4 hours apart after 20 weeks of gestation, except molar pregnancies [1], and proteinuria: $0.3 \mathrm{~g}$ or greater after a 24 -hour urine sample [2].

The pathogenesis of $\mathrm{PE}$ is not completely understood. It is thought to involve: defective placentation, diminished perfusion of placenta, hypoxia, increased oxidative stress, production of lipid-laden foam cells as seen in atherosis, release of antiangiogenic proteins into the maternal blood, excessive maternal inflammatory response, endothelial injury, generalized endothelial dysfunction, hypertension, activation of the coagulation cascade, multiorganic manifestations [3-13].

Pre-eclampsia was classified in early-onset (clinical onset of $\mathrm{PE}<34$ weeks of gestation) and late-onset (PE at $\geq 34$ weeks of gestation) $[6,12]$. There is also a hereditary predisposition [14]. The familial susceptibility to the development of pre-eclampsia may be mediated by single nucleotide polymorphisms in the Toll-like receptors gene sequences and can be associated with early-onset PE [15]. There are studies that indicate an association between pre-eclampsia and chronic infectious diseases $[8,16,17]$.

* Correspondence to: Carmen Rădulescu

E-mail: carmenc.radulescu@yahoo.com
Chronic infection-associated inflammation may trigger maternal response like pre-eclampsia $[8,9,16]$.

Cytomegalovirus (CMV) is a Deoxyribonucleic acid (DNA) herpesvirus. It can be transmitted by contact with infected nasopharyngeal secretions, urine, saliva, semen, cervical secretions, blood. There may be intrauterine, intrapartum or neonatal infection from breast feeding. Up to 85 percent of women from lower socioeconomic backgrounds are seropositive by the time of pregnancy whereas only half of women in higher income groups are immune. Maternal antibodies do not prevent fetal infection [18]. Some seropositive women can be reinfected with a different strain of virus $[18,19]$. Infection with cytomegalovirus impairs extravillous cytotrophoblasts migration and efficient invasion of uterine wall and maternal spiral arteries induced by chemokine CXCL12 [10].

In our study we investigated if there is a potential link between cytomegalovirus infection and pre-eclampsia. It was estimated about 50,000 maternal deaths per year worldwide because of pre-eclampsia [20] and it is important an intensive surveillance to avoid adverse outcomes in $\mathrm{PE}$ and fetal or maternal demise.

\section{Material and methods}

This study was carried out between January 2014 to July 2015 at the Obstetrics and Gynecology Department in Târgu Mureş University Hospital, Romania. Our Hospital and University Ethics Committee approved the study. The purpose of our study was explained to our patients 
and a written informed consent was obtained from each participating woman. We included two women groups: 66 with pre-eclampsia and 62 without pre-eclampsia (controls, normotensive pregnant women). The blood pressure was taken in a semi-recumbent position, with a supported arm and appropriately-sized cuff using a manual sphygmomanometer, with Korotkoff $\mathrm{V}$ used to determine diastolic pressure.

Maternal venous blood samples $(5 \mathrm{ml})$ were collected into vacutainer tubes without anticoagulants. After sample collection, immediately serum was separated by centrifugation and stored at $-80^{\circ} \mathrm{C}$ until assayed at the Central Laboratory of Mureş County Emergency Hospital. Enzymelinked immunosorbent assay (ELISA) was performed to detect cytomegalovirus Immunoglobulin G (Dia. Pro CMV IgG kit, ELISA, Italy) in maternal serum samples according to the manufacturers' instructions. A calibration curve, calibrated against the $1^{\text {st }}$ World Health Organisation (WHO) international standard, made possible a quantitative determination of the antibody in the patient. The cutoff value for cytomegalovirus Immunoglobulin G (IgG) was of $0.5 \mathrm{IU} / \mathrm{ml}$. Samples with a concentration lower than $0.5 \mathrm{WHO} \mathrm{IU} / \mathrm{ml}$ were considered negative for anti Cytomegalovirus IgG antibody. Samples with a concentration higher than $0.5 \mathrm{WHO} \mathrm{IU} / \mathrm{ml}$ were considered positive for anti Cytomegalovirus IgG antibody.

Statistical data were analyzed using SPSS 17.0 for Windows program.

To characterize the two groups of patients we used mean, standard deviation, the minimum and maximum values recorded in the study. We used one sample Kolmogorov-Smirnov test for evaluation the significance of average values of continuous variables, the socio-demographic variables and the levels of cytomegalovirus IgG.

Chi-squared test was used for categorical variables. Data were reported as mean \pm standard deviation and percentage for continuous demographic characteristics, median and range for the levels of cytomegalovirus IgG. We used Mann-Whitney $U$ tests to compare the cytomegalovirus IgG levels of preeclamptic group and for controls. A $p$ value $<0.05$ was considered significant for all tests.

We also estimated the risk and 95\% confidence interval (CI) of cytomegalovirus IgG in pre-eclamptic women and controls using SPSS 17.0 soft.

\section{Results}

In table I are shown the maternal clinical characteristics and p-value of the 66 pre-eclamptic women and of the 62 healthy pregnancy control: age (years), the percentage of nulliparous women, the gestational age at delivery (in weeks), the mean systolic blood pressure $(\mathrm{mmHg})$, the mean diastolic blood pressure $(\mathrm{mmHg})$, the percentage of proteinuria, the birth weight $(\mathrm{g})$, the percentage of smokers, body mass index (BMI) before pregnancy $\left(\mathrm{kg} / \mathrm{m}^{2}\right)$, the current BMI. Pre-eclamptic women had a higher mean blood pressure and a current BMI higher than controls.

The mean triglycerides values were of $266 \mathrm{mg} / \mathrm{dl}$ in PE group and of $209 \mathrm{mg} / \mathrm{dl}$ in the controls. In PE group, $77 \%$ had a family history of hypertension and $62 \%$ in the control group . In preeclamptic group $32 \%$ had a family history of diabetes and 19\% in the control group. In PE group 23\% had a family history of obesity and 19\% in the control group.

As shown in table II, the median value of cytomegalovirus IgG in pre-eclamptic women is increased: $4.573 \mathrm{IU} / \mathrm{ml}$ with a minimum value of 0.00 and a maximum value of $71.3 \mathrm{IU} / \mathrm{ml}$ compared with the median value in the control group: $4.066 \mathrm{IU} / \mathrm{ml}$ with a minimum value of 0.00 and a maximum value of $304 \mathrm{IU} / \mathrm{ml}$, but p-value: 0.867 (not significant) in Mann-Whitney $\mathrm{U}$ test.

The odds ratio (OR) for qualitative cytomegalovirus IgG (table III) was of 0.933 (95\%CI:0.284-3.064). The OR for CMV IgG in pre-eclamptic group was of 0.967 (95\% CI: $0.535-1.748)$ which do not differ greatly from the value recorded in the control group OR: .036 (95\%CI: 0.571-1.880)

The pathophysiology of pre-eclampsia is believed to involve impaired placentation and an associated increase in systemic inflammation. In our study we have determined the white blood cells and the neutrophils percentage in pre-eclamptic women and in healthy pregnant women in order to confirm or infirm the hypothesis that cytomegalovirus infection produces a generalized inflammation and endothelial injury, pathogenic features of pre-eclampsia (Table IV).

Table IV shows that in PE group the median value of white blood cells (WBC) was of $11.645 \times 10^{3} / \mu \mathrm{L}$ with a minimum value of $5 \times 10^{3}$ and a maximum value of $21.1 \times 10^{3}$, higher than in the control group: $9.42 \times 10^{3} / \mu \mathrm{L}$

Table I. Maternal and perinatal clinical characteristics in women with pre-eclampsia and in normal pregnancy control group

\begin{tabular}{|c|c|c|c|}
\hline Characteristic & Pre-eclampsia $(\mathrm{n}=66)$ & Normal pregnancy control $(\mathrm{n}=62)$ & P-value \\
\hline Maternal age (years) & $28 \pm 6.3(14-44)$ & $26 \pm 7(14-42)$ & $0.120^{*}$ \\
\hline Nulliparous (\%) & 75 (l, multi) & 79.4 (I, multi) & $0.100^{*}$ \\
\hline Gestational age at delivery (weeks) & $37.7 \pm 2.3(31-41)$ & $38 \pm 5.7(38.5-41)$ & $0.173^{*}$ \\
\hline Mean systolic blood pressure $(\mathrm{mmHg})$ & $152.9 \pm 17.9$ & $114 \pm 7.4$ & $0.000^{*}$ \\
\hline Mean diastolic blood pressure $(\mathrm{mmHg})$ & $97.15 \pm 11.1$ & $70.6 \pm 5.5$ & $0.000^{*}$ \\
\hline Proteinuria (\%) & 100 & 0 & $0.000^{*}$ \\
\hline Birth weight $(\mathrm{g})$ & $3076 \pm 714(1570-4730)$ & $3218 \pm 485(1730-4280)$ & $0.093^{\star *}$ \\
\hline Smoker (\%) & 73.5 & 79.4 & $0.500^{*}$ \\
\hline BMI before pregnancy & $27.3 \pm 6.8(15.6-48.1)$ & $21.1 \pm 2.8(18-29)$ & $0.019^{* *}$ \\
\hline Current BMI & $31.04 \pm 6.6(20.7-52.5)$ & $25.7 \pm 4(20-41.5)$ & $0.004^{\star *}$ \\
\hline
\end{tabular}


Table II. Median and range for CMV IgG in PE and in the control pregnant women

\begin{tabular}{cccc}
\hline Characteristic & $\begin{array}{c}\text { Pre-eclampsia } \\
(\mathrm{n}=66)\end{array}$ & $\begin{array}{c}\text { Normal pregnancy control } \\
(\mathrm{n}=62)\end{array}$ & $\begin{array}{c}\text { P-value } \\
(\text { Mann-Whitney U test) }\end{array}$ \\
\hline CMV IgG IU/ml (median) & $4.573(0.00-71.3)$ & $4.066(0.00-304)$ \\
\hline CMV: Cytomegalovirus; IgG: Immunoglobulin G; PE: Preeclamptic & & 0.867 \\
\hline
\end{tabular}

Table III. The estimated risk of CMV IgG in PE women and controls

\begin{tabular}{lcc}
\hline & \multicolumn{2}{c}{$95 \%$ Confidence Interval } \\
\cline { 2 - 3 } & Value & Lower \\
\hline Odds Ratio for qualitative CMV IgG (negative $<0.5 \mathrm{IU} / \mathrm{ml} /$ positive over 0.5 IU/ml) & 0.933 & 0.284 \\
For PE cohort & 0.967 & 0.535 \\
For control cohort & 1.036 & 0.571 \\
Number of Valid Cases & 1.748 & 1.880 \\
\hline
\end{tabular}

Table IV. Median and range of white blood cells and neutrophils in PE and in control group

\begin{tabular}{|c|c|c|c|}
\hline Characteristic & Pre-eclampsia $(\mathrm{n}=66)$ & Normal pregnancy control $(n=62)$ & P-value (Kolmogorov-Smirnov test) \\
\hline White blood cells $(/ \mu \mathrm{L})$ (median) & $11.645 \times 10^{3}\left(5 \times 10^{3}-21.1 \times 10^{3}\right)$ & $9.42 \times 10^{3}\left(4.67 \times 10^{3}-18.4 \times 10^{3}\right)$ & 0.000 \\
\hline Neutrophils (\%) (median) & $77.7 \%(57.5 \%-93.7 \%)$ & $69.2 \%(52.38 \%-87 \%)$ & 0.000 \\
\hline
\end{tabular}

with a minimum value of $4.67 \times 10^{3}$ and a maximum value of $18.4 \times 10^{3}$ and $\mathrm{p}$-value was significant $(\mathrm{p}=0.000)$.

In pre-eclamptic group we have found a positive directly correlation (Pearson correlation coefficient: 0.217) with a significant "p" value of 0.08 between cytomegalovirus IgG values and the number of white blood cells in $92 \%$ of women. In pre-eclamptic group Pearson correlation coefficient was negative between cytomegalovirus IgG value and the neutrophils percentage.

In normotensive pregnant women (controls) we did not find a statistically significant correlation between cytomegalovirus IgG and WBC count and also between cytomegalovirus IgG and the percentage of neutrophils.

We had determined pro-inflammatory cytokines: interleukine (IL) 6 and 16 between 35 and 40 weeks of gestation for a subgroup of 68 pregnant women (47 PE and 21 controls) but we did not find significant correlation between IL-6, IL-16, cytomegalovirus IgG infection in pre-eclamptic and in the control group; Pearson correlation coefficient was negative: -0.097 and a $p$-value of 0.524 between IL- 6 and cytomegalovirus IgG and -0.63 and a p -value of 0.679 between IL-16 and cytomegalovirus IgG.

We had also calculated (Table V and table VI) if there is a greater risk in pregnant women with pre-eclampsia and positive cytomegalovirus IgG for premature birth and those at term delivery. So we have recorded 23 of $26 \mathrm{PE}$ patients with values of cytomegalovirus $\mathrm{IgG}$ over $0.5 \mathrm{IU} / \mathrm{ml}$ which delivered premature and 35 of $40 \mathrm{PE}$ patients with positive cytomegalovirus IgG which delivered at term. According to table VI data, odds ratio for cytomegalovirus IgG in pre-eclamptic women which delivered premature and in those which had at term delivery was of 1.014 (OR:1.014; 95\%CI:0.157-6.549). The risk in pre-eclamptic women with CMV IgG values of over $0.5 \mathrm{IU} / \mathrm{ml}$ which delivered premature was greater (OR:1.009; 95\%CI:0.329-3.090) than those which delivered at term (OR:0.994; 95\%CI: $0.472-2.095)$ but the differences between the two values were not very high.

\section{Discussion}

It is important to avoid adverse outcomes in pre-eclampsia such as maternal and fetal demise. A major goal of our study was to identify women at high risk of PE, to search if cytomegalovirus infection may influence the onset of preeclampsia.

Cytomegalovirus infection can increase in the host immune response and release chemokines and cytokine responses: Interferon gamma (IFN- $\gamma$ ), Tumour necrosis factor alpha (TNF- $\alpha$ ), Interleukines 4,8,18 (IL-4, IL-8, IL-18), modifies adhesion molecules: Intercellular adhesion molecule-1 (ICAM-1), Vascular cell adhesion molecule-1 (VCAM-1), E-selectin, increases matrix metallopro-

Table V. CMV IgG, gestational age, premature, at term delivery crosstabulation

\begin{tabular}{|c|c|c|c|c|}
\hline & & \multicolumn{2}{|c|}{ Gestational age, premature, at term } & \multirow{2}{*}{ Total } \\
\hline & & premature $<38$ weeks & at term $38-41$ weeks & \\
\hline \multirow{3}{*}{ CMV IgG } & negative $<0.5 \mathrm{IU} / \mathrm{ml}$ & 3 & 5 & 8 \\
\hline & positive $>0.5 \mathrm{IU} / \mathrm{ml}$ & 23 & 35 & 58 \\
\hline & & 26 & 40 & 66 \\
\hline
\end{tabular}

Table VI. Risk estimate for premature vs. at term delivery in CMV IgG seropositive women

\begin{tabular}{|c|c|c|c|}
\hline & \multirow[b]{2}{*}{ Value } & \multicolumn{2}{|c|}{ 95\% Confidence Interval } \\
\hline & & Lower & Upper \\
\hline Odds Ratio for CMV IgG(negative $<0.5 \mathrm{IU} / \mathrm{ml} /$ positive over $0.5 \mathrm{IU} / \mathrm{ml}$ ) & 1.014 & 0.157 & 6.549 \\
\hline For cohort premature $<38$ weeks & 1.009 & 0.329 & 3.090 \\
\hline $\mathrm{N}$ of valid cases & 66 & & \\
\hline
\end{tabular}


teinase (MMP-2) activity, resulting an alteration in vessel remodeling, enhance the adherence and infiltration of inflammatory cells that drive vascular disease. These pathophysiological mechanisms were found in atherosclerosis, transplant vascular sclerosis, vessel narrowing and acute organ transplant rejection [21]. In our study the mean triglycerides values were higher in PE group $(266 \mathrm{mg} / \mathrm{dl})$ than in the controls $(209 \mathrm{mg} / \mathrm{dl})$. Women who had preeclampsia more frequently develop cardiovascular disease later in life $[22,23]$. We had determined pro-inflammatory cytokines, interleukine (IL) 6 and 16 between 35 and 40 weeks of gestation for a subgroup of 68 pregnant women (47 pre-eclamptic and 21 controls) but we did not find a statistically significant correlation between IL-6, IL-16, cytomegalovirus IgG infection in pre-eclamptic and control group; may be the cause was our sample size relatively small.

Xie $\mathrm{F}$ et al. [9] studied cytomegalovirus Immunoglobulin $\mathrm{G}, \mathrm{M}$ and $\mathrm{A}$ (IgG, IgM, IgA) seropositivity in pre-eclamptic and normal pregnancy controls. They found that cytomegalovirus IgG antibody level was higher in PE than in normal controls $(\mathrm{p}<0.0001)$ but no difference was observed in cytomegalovirus IgM or IgA among study groups. IgA is associated with primary, chronic, or recurrent infections and IgM suggests acute or primary infection. The authors concluded that cytomegalovirus infection may influence the occurrence of pre-eclampsia because cytomegalovirus infection can activate inflammatory cytokine responses via CD14 and Toll-like receptor 2. No infants had clinical evidence of congenital cytomegalovirus.

Rustveld LO et al. [16] collected data of published studies since 1964 on relationship between maternal infection and pre-eclampsia. The authors selected sixteen of 32 studies. They calculated OR (odds ratio), 95\% CI and found in their analysis that any infection (bacterial or viral) was associated with a two-fold higher risk of pre-eclampsia. The association may provide a potential explanation for preeclampsia-related inflammation.

Conde Agudelo A et al. [8] examined the relationship between maternal infection and pre-eclampsia from fortynine studies. They ascertained that there were no association between pre-eclampsia and presence of antibodies to Chlamydia pneumoniae, Helicobacter pylori, Cytomegalovirus, Human Immunodeficiency Virus (HIV) infection, malaria, herpes simplex virus type 2 , bacterial vaginosis and Mycoplasma hominis. The authors found that the risk of pre-eclampsia was increased in pregnant women with urinary tract infection (pooled OR:1.57; 95\%CI:1.451.70 ) and periodontal disease (pooled OR:1.76; 95\%CI: 1.43-2.18).

Gibson CS et al. [17] had investigated the role of fetal exposure to herpesvirus infection and pregnancy-induced hypertensive disorders, antepartum haemorrhage, birth weight $<10$ th percentile, preterm birth, using polymerase chain reaction. They concluded that fetal exposure to herpesvirus infection was associated with pregnancy-induced hypertensive disorders for term and preterm births. Cytomegalovirus belongs to herpesvirus family.

Strand KM et al. [24] studied cytomegalovirus IgG and IgM in plasma sample of a cohort of 1500 women with pre-eclampsia and 1500 healthy pregnant women. They concluded that maternal antibodies to cytomegalovirus was not associated with pre-eclampsia; their result suggested that cytomegalovirus infection was unlikely be a major cause of PE.

The results of our study suggest that the median value cytomegalovirus IgG seropositivity in pre-eclamptic women was increased compared with the median value in the control group but "p" value was not significant. IgG cytomegalovirus indicates previous infection. When we had calculated the odds ratio for cytomegalovirus IgG in preeclamptic group we evaluated that it did not differ greatly from the value recorded in the control group. When we studied the risk for preterm birth in pre-eclamptic women CMV IgG positive compared to those who delivered at term, the differences between the two values were not very high. We did not find a correlation coefficient between the percentage of neutrophils and cytomegalovirus IgG infection in pre-eclamptic women.

There are some limitations of this study. Our sample size was relatively small and we did not studied cytomegalovirus IgA which is associated with primary, chronic, or recurrent infection nor IgM which indicate acute or primary cytomegalovirus infection neither CMV-DNA plasma viral load, PP65 antigen; however Xie F et al. [9] did not observed any difference between IgM or IgA levels in their study.

It is necessary a larger number of patients to conclude that cytomegalovirus infection can influence the onset of pre-eclampsia.

In our study, no infants exhibited clinical evidence of congenital CMV infection.

\section{Conclusions}

Preeclampsia is an important cause of maternal and neonatal morbidity and mortality. The pathogenesis of PE is not clear; some studies found an association between chronic infection-associated inflammation and pre-eclampsia. In our study, we concluded that cytomegalovirus infection is unlikely to be the triggering factor of pre-eclampsia. More studies are required to verify on a greater number of pregnant women if there is any relationship between cytomegalovirus infection and pre-eclampsia.

\section{Acknowledgement}

This study was supported by an internal grant (nr. 16441/11.12.2013) of University of Medicine and Pharmacy of Târgu Mureş, Romania.

\section{Conflicts of interest}

The authors report no conflicts of interest. 


\section{Abbreviation}

Interferon gamma (IFN- $\gamma$ ), Tumour necrosis factor alpha (TNF $\alpha$ ), Intercellular adhesion molecule-1 (ICAM-1), Vascular cell adhesion molecule1 (VCAM-1), Interleukin 8 (IL-8), Body mass index (BMI)

\section{References}

1. Espinoza J, Uckele JE, Starr RA, et al. Angiogenic imbalances: the obstetric perspective. Am J Obstet Gynecol 2010;203(1):17.e1-8.

2. ACOG Committee on Obstetric Practice. ACOG Practice Bulletin Diagnosis and management of preeclampsia and eclampsia. Number 33. January 2002. American College of Obstetricians and Gynecologists. Int J Gynecol Obstet 2002;77(1):67-75

3. Rasmussen LG, Lykke JA, Staff AC. Angiogenic biomarkers in pregnancy:defining maternal and fetal health. Acta Obstet Gynecol Scand 2015;94(8):820-32.

4. Staff AC, Dechend R, Redman CW. Review: Preeclampsia, acute atherosis of the spiral arteries and future cardiovascular disease: two new hypothese. Placenta 2013;34:S73-8.

5. Staff AC, Johnsen GM, Dechend R, Redman CWG. Preeclampsia and uteroplacental acute atherosis: immune and inflammatory factors. J Reprod Immunol 2014;101-102:120-26.

6. Redman CW, Sargent il, Staff AC. IFPA Senior award lecture: making sense of pre-eclampsia-Two placental causes of preeclampsia? Placenta 2014:35:S20-S25

7. Macey MG, Bevan S, Alam S et al. Platelet activation and endogenous thrombin potential in pre-eclampsia. Thromb Res 2010;125(3):e76-81.

8. Conde-Agudelo A, Vilar J, Lindheimer M. Maternal infection and risk of preeclampsia: systemic review and metaanalysis. Am J Obstet Gynecol 2008;198(1):7-22

9. Xie F, Hu Y, Magee Laura A, et al. An association between cytomegalovirus infection and pre-eclampsia: a case-control study and data synthesis. Acta Obstet Gynecol Scand 2010;89(9):1162-7.

10. Warner JA, Zwezdaryk KJ, Day B, Sullivan DE, Pridjian G, Morris CA. Human cytomegalovirus infection inhibits CXCL12-mediated migration and invasion of human extravillous cytotrophoblasts. Virol J. 2012;1(9):255.

11. Fisher SJ. Why is placentation abnormal in preeclampsia? Am J Obstet
Gynecol 2015; 213(4Suppl):S115-22.

12. Xie F, von Dadelszen P, Nadeau J. CMV infection, TLR-2 and-4 expression, and cytokine profiles in early-onset preeclampsia with HELLP syndrome. Am J Reprod Immunol 2014;71(4):379-86.

13. Xie F, Turvey SE, Williams MA, Mor G, von Dadelszen P. Toll-like receptor signaling and pre-eclampsia. Am J Reprod Immunol 2010;63(1):7-16.

14. Cunningham GF, Leveno KJ, Bloom SL,et al. Chapter 40: Hypertensive disorders in Williams Obstetrics 24rd Edition, McGrawHill Med Ed. USA. 2014:728-79.

15. Xie F, Hu Y, Speert DP, et al. Toll-like receptor gene polymorphisms and preeclampsia risk: a case- control study and data synthesis. Hypertens Pregnancy 2010;29(4):390-8.

16. Rustveld LO, Kelsey SF, Sharma R. Association between materna infections and preeclampsia: a systematic review of epidemiologic studies. Matern Child Health J 2008;12(2):223-42.

17. Gibson CS, Goldwater PN, MacLennan AH, et al. South Australian Cerebral Palsy Research Group. Fetal exposure to herpesviruses may be associated with pregnancy-induced hypertensive disorders and preterm birth in a Caucasian population. BJOG 2008;115(4):492-500.

18. Cunningham GF, Leveno KJ, Bloom SL, et al. Chapter 64: Infectious diseases. Cytomegalovirus in Williams Obstetrics 24rd Edition, McGrawHill Med Ed. USA. 2014:1245-48.

19. Boppana SB, Rivera LB, Fowler Kbet al. Intrauterine transmission of cytomegalovirus to infants of women with preconceptional immunity. N Engl J Med 2001;344(18):1366-71

20. Norwitz ER, Funai EF. Expectant management of severe preeclampsia remote from term: hope for the best, but expect the worst. Am J Obstet Gynecol 2008;199(3):209-12.

21. Streblow DN, Dumortier J, Moses AV, Orloff SL, Nelson JA. Mechanisms of cytomegalovirus-accelerated vascular disease: induction of paracrine factors that promote angiogenesis and wound healing. Curr Top Microbiol Immunol 2008;325:397-415.

22. Bellamy L, Casas JP, Hingorani AD, Williams DJ. Pre-eclampsia and risk of cardiovscular disease and cancer in later life: systematic review and meta-analysis. BMJ 2007;335(7627):974.

23. Xie F, Hu F, Turvey SE et al. Toll-like receptors 2 and 4 and cryopyrin inflammasome in normal pregnancy and pre-eclampsia. BJOG 2010;117(1):99-108.

24. Strand KM, Odland ML, Iversen AC, et al. Cytomegalovirus antibody status at 17-18 weeks of gestation and pre-eclampsia: a case-control study of pregnant women in Norway. BJOG 2012;119(11):1316-23. 